\title{
Empregando Análise Visual e Sensemaking no Ensino de Predictive Suffix Trees
}

\author{
Antonio José M. Leite Júnior ${ }^{1}$, Emanuele Santos ${ }^{1}$, Creto A. Vidal ${ }^{1}$, Cleilton Lima Rocha ${ }^{2}$ \\ ${ }^{1}$ Universidade Federal do Ceará \\ ${ }^{2}$ Instituto Atlântico - Fortaleza/Ceará \\ melojrevirtual.ufc.br, \{emanuele,cvidal\}@dc.ufc.br, \\ cleilton_rochaeatlantico.com.br
}

\begin{abstract}
Teaching abstract concepts can be quite complicated. In this sense, understanding Predictive Suffix Trees (PSTs) is particularly challenging since this type of tree-based data structure simultaneously stores and relates information about space, time, and probabilities. This work presents a practical experiment that tries to make the teaching of PSTs more applied, based on the development of a specific application, which is tested and analyzed quantitatively and qualitatively, demonstrating that the proposed solution can be a viable educational alternative.
\end{abstract}

Resumo. O ensino de conceitos abstratos pode ser bastante complexo. Nesse sentido, compreender Predictive Suffix Trees (PSTs) é particularmente desafiador, uma vez que esse tipo de estrutura de dados, baseada em árvores, armazena e relaciona simultaneamente informações sobre espaço, tempo e probabilidades. Esse trabalho apresenta um experimento prático que visa tornar mais aplicado o ensino de PSTs, baseando-se no desenvolvimento de uma aplicação específica, que é testada e analisada quantitativa e qualitativamente, demonstrando que a solução proposta pode ser uma alternativa educacional viável.

\section{Introdução}

Recentemente, [Rocha et al. 2016] propuseram um modelo preditivo para indicar quando indivíduos poderiam deixar suas posições atuais para ir a um novo local, usando PSTs Probabilistic Suffix Trees (Figura 1), estruturas de dados, baseadas na ideia de árvores, capazes de representar simultaneamente espaço, tempo e probabilidade [Ron et al. 1994].

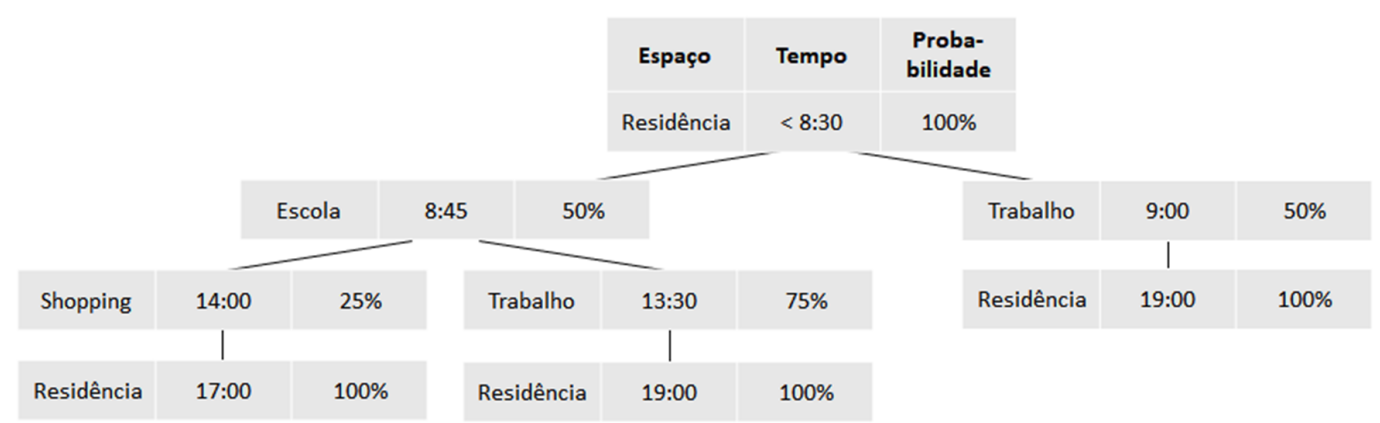

Figura 1. Exemplo simples de Predictive Suffix Tree. 
VII Congresso Brasileiro de Informática na Educação (CBIE 2018)

Anais do XXIX Simpósio Brasileiro de Informática na Educação (SBIE 2018)

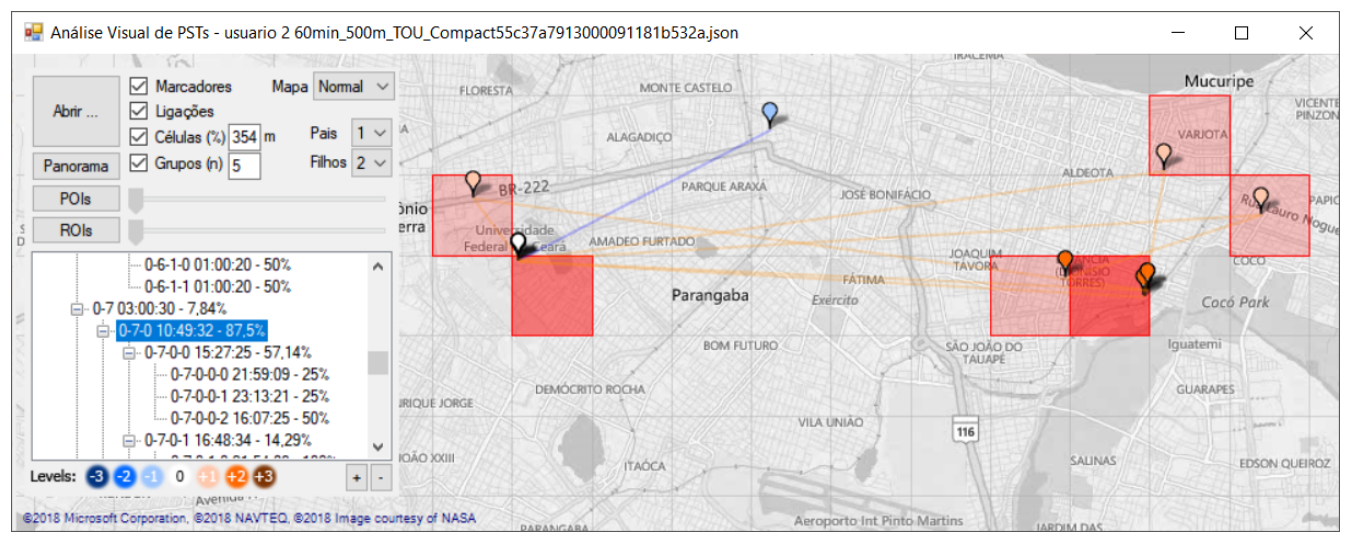

Figura 2. Visualização de uma PST empregando a ferramenta desenvolvida.

Posteriormente, empregando o conceito de análise visual, [Leite et al. 2017] adaptaram esse modelo para definir uma solução que auxiliasse na identificação de padrões de comportamentos de indivíduos. Isso permitiu investigar, por exemplo, onde uma pessoa possivelmente residia ou provavelmente onde e quando ela trabalhava ou estudava. Esse tipo de proposta visa contribuir para a substituição do método tradicional de avaliação de PSTs, baseado na interpretação de linhas de texto repletas de dados de latitudes, longitudes, datas, horários e porcentagens, por uma solução interativa, simplificando a compreensão das informações representadas e suas interrelações.

Complementando os esforços de [Leite et al. 2017], o presente trabalho propõe a adoção de sensemaking como forma de simplificar o ensino de PSTs, buscando tornar mais prática a exposição desse conceito que é considerado muito abstrato por alunos e professores [Ron et al. 1994]. Para tanto, foi desenvolvida uma aplicação específica (Figura 2), que foi testada por alunos de graduação a fim de se avaliar possíveis ganhos educacionais e as análises quantitativa e qualitativa dos resultados demonstraram a viabilidade da solução proposta. O restante desse trabalho encontra-se organizado em mais seis seções. A Seção 2 apresenta trabalhos relacionados. Conceitos básicos são discutidos na Seção 3. A aplicação desenvolvida é apresentada na Seção 4 e avaliada pelo experimento apresentado na Seção 5. Os resultados obtidos são analisados na Seção 6 e, por fim, a Seção 7 expõe as considerações finais sobre esse trabalho.

\section{Trabalhos Relacionados}

Ainda não é comum a adoção da análise visual como ferramenta para o ensino de conceitos abstratos [Siemens and Long 2011]. No entanto, uma série recente de trabalhos tem buscado aliar a análise visual à metodologia de sensemaking, facilitando processos de aprendizagem em diversos graus. Por exemplo, [Caglayan 2018] avalia como o uso do software MATLAB ${ }^{1}$, incluindo a geração de imagens, pode ser adotado por alunos universitários no estudo de conceitos de álgebra linear. Já [Bobis and Way 2018] discutem como diferentes meios de representação empregados por crianças, incluindo suas produções digitais, podem ser explorados no ensino de matemática. Na mesma área, porém adotando tecnologias mais recentes, [ $\mathrm{Ng}$ et al. 2018] descrevem como interações, mediadas por software, entre modelos tridimensionais virtuais e concretos podem ser uma alternativa

\footnotetext{
${ }^{1}$ http://www.mathworks.com/products/matlab.html
} 
VII Congresso Brasileiro de Informática na Educação (CBIE 2018)

Anais do XXIX Simpósio Brasileiro de Informática na Educação (SBIE 2018)

no ensino de geometria e de trigonometria. No campo da física, [Beheshti et al. 2018] utilizam mesas interativas num museu para apresentar conceitos básicos de circuitos eletrônicos a pais e filhos. E [Warburton and Laughlin 2018] vão além, e exploram a fusão entre dança e imagens digitais projetadas num palco buscando facilitar a interpretação de problemas relacionados à Lei da Gravitação Universal de Newton.

\section{Conceitos Básicos}

Nessa seção são apresentados três conceitos importantes para a correta compreensão desse trabalho: análise visual, sensemaking e branching time.

\subsection{Análise Visual}

Análise visual consiste num conjunto de métodos, tecnologias e práticas que combinam as capacidades de raciocínio, intrínsecas a seres humanos, e o processamento de dados por máquinas [Keim et al. 2008]. É geralmente considerada diferente de outras abordagens investigativas (data-mining ou visualização científica, por exemplo) por se basear em representações interativas, que ampliam as capacidades humanas de detectar padrões, estabelecer relações ou criar inferências [Fabrikant and Lobben 2009]. Assim, o principal objetivo da análise visual é permitir que, empregando software específico, analistas superem suas capacidades naturais de interpretação, sendo os resultados obtidos supostamente menos limitados que aqueles obtidos exclusivamente por procedimentos automáticos, pois os usuários podem usar seus conhecimentos prévios para elaborar perguntas (estabelecendo hipóteses) e procurar respostas (confirmando ou refutando as hipóteses estabelecidas), à medida que observam mudanças nos eventos e nos contextos avaliados [Fabrikant and Lobben 2009]. De forma complementar, a análise visual pode fazer uso de ferramentas automáticas ou semiautomáticas para ajudar usuários a realizarem investigações mais rápidas e eficientes.

\subsection{Sensemaking}

Apesar de muitas de suas ideias já terem sido empregadas desde os primeiros experimentos que visavam auxiliar a interação entre humanos e computadores, a metodologia sensemaking só foi formalmente estabelecida na ciência da computação na década de 1980 [Dervin 1983]. Essa metodologia, que significa "dar sentido", consiste na simplificação de representações de informações para facilitar a execução de tarefas, sendo uma prática interdisciplinar que se apropria e estende conceitos oriundos de vários campos do conhecimento, como educação, psicologia social, filosofia e sociologia, por exemplo [Russell et al. 1993]. Sensemaking é bastante aplicada na criação de interfaces gráficas, principalmente mudando contextos de uso para empregar conhecimentos prévios de usuários. Como exemplo prático, pode-se citar a ação de jogar um documento na lata de lixo virtual para apagar o respectivo arquivo no computador, presente na hoje tão comum metáfora de desktop (área de trabalho) [Blackwell 2006]. Nesse caso, o usuário utiliza conceitos e conhecimentos de seu mundo concreto e cotidiano (documento e lata de lixo) para realizar operações no computador, substituindo a aprendizagem de ideias abstratas (arquivo) e de comandos textuais para lidar com o sistema operacional.

\subsection{Branching Time}

Inicialmente, o termo branching time relacionava-se à classificação de estruturas narrativas presentes em algumas obras literárias, quando um dado evento podia dar origem a 
VII Congresso Brasileiro de Informática na Educação (CBIE 2018)

Anais do XXIX Simpósio Brasileiro de Informática na Educação (SBIE 2018)

dois ou mais eventos distintos [Martínez 2011]. Posteriormente, considerando a evolução dos meios de comunicação, o branching time passou a ser aplicado também em outras mídias, como filmes, peças teatrais e jogos eletrônicos, por exemplo. Hoje em dia esse conceito tem se tornado particularmente importante para investigar implicações de uma ação sobre possibilidades futuras, auxiliando na interpretação de cenários alternativos e no processo de tomada de decisões como um todo [Aigner et al. 2008].

\section{Aplicação Desenvolvida}

Para avaliar a união de análise visual, sensemaking e branching time foi desenvolvida uma aplicação de visualização interativa (Figura 2) que relaciona diretamente os três componentes de análise considerados (espaço, tempo e probabilidades), permitindo a exploração de dados de uma PST para a descoberta de padrões prováveis de comportamento de indivíduos. A aplicação usa dados produzidos pelo Projeto Eaí? ${ }^{2}$, um aplicativo para dispositivos móveis que fornece o cardápio diário do restaurante universitário da Universidade Federal do Ceará, contando com aproximadamente dois mil usuários ativos. Sem qualquer tipo de identificação por motivos de privacidade, as posições de GPS desses usuários e seus respectivos tempos associados são capturados pelos dispositivos móveis e enviados ao servidor do Projeto, que processa os dados e gera PSTs que são armazenadas em arquivos JSON.

A aplicação lê os arquivos JSON, extraindo os dados armazenados (coordenadas geográficas, informações temporais e probabilidades associadas), organiza os nós da PST numa tree list, preservando todos os níveis da árvore, e apresenta-os num mapa geográfico. Clicando diretamente numa linha da tree list, é possível selecionar o respectivo nó da PST e exibir automaticamente um marcador no mapa de acordo com as coordenadas geográficas do mesmo. Caso esse nó possua antecessores (nós pais) ou sucessores (nós filhos), seus respectivos marcadores também podem ser mostrados, diferenciando-os de acordo com seus níveis na PST. Marcadores indicando nós pais e filhos são coloridos usando uma escala cromática complementar, variando de azul escuro para o nó pai de terceiro nível até laranja escuro para o nó filho de terceiro nível. É possível ainda apresentar no mapa as ligações, que são projeções das arestas da PST que conectam o nó selecionado aos seus nós pais e filhos, seguindo a mesma escala cromática. Apropriando-se do conceito de branching time, esses nós pais e filhos representam respectivamente situações que ocorreram antes e depois do nó selecionado na tree list, com as ligações então representando graficamente as relações temporais entre tais situações. De forma complementar, também se pode realizar o processo de seleção inversa, clicando num marcador no mapa e automaticamente selecionando sua respectiva linha na tree list.

Ao se definir o tamanho de uma grade visual, dados podem ser acumulados para explorar segmentos espaciais no mapa, surgindo grupos quadrados cujos tons de vermelho são proporcionais ao número de marcadores presentes em cada célula (Figura 3a). Pode-se também estabelecer um limite máximo para a contagem de ocorrências, controlando o cálculo desses tons de vermelho. Se a grade visual estiver desativada, ao redor dos marcadores são apresentadas células que representam as probabilidades de cada nó individualmente (Figura 3b). Esses grupos e células podem servir de base para a identificação rápida de pontos de interesse (POIs, points of interest) para aprofundamento

\footnotetext{
${ }^{2} \mathrm{http}: / /$ www.appeai.com
} 


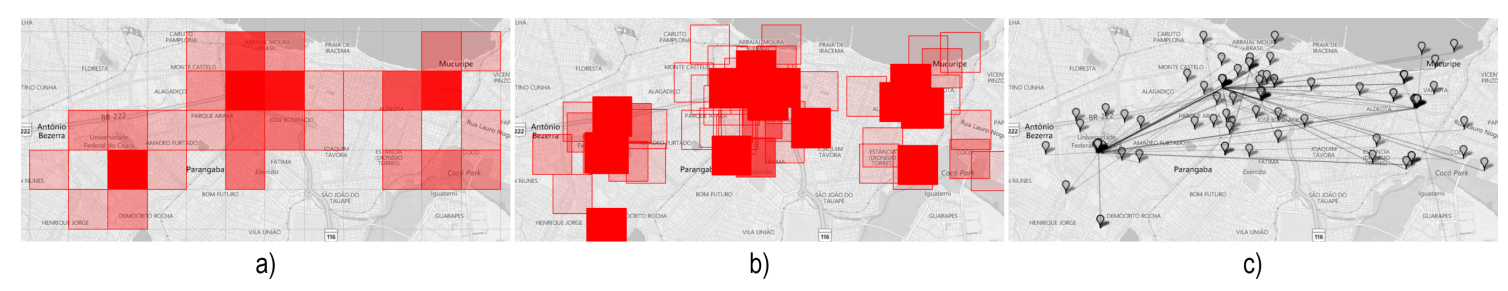

Figura 3. Exemplos de (a) grupos, (b) células e (c) panorama.

de investigações. Também é possível apresentar panoramas (Figura 3c), que exibem todos os dados armazenados numa PST simultaneamente no mapa, podendo-se exibir ou ocultar individualmente marcadores, ligações, células e grupos.

Opcionalmente, pode-se ainda utilizar funções semiautomáticas para identificação de POIs e de rotas de interesse (ROIs, routes of interest), que contabilizam respectivamente o conjunto de marcadores (Figura 4a) e de ligações (Figura 4b) do mapa e, de acordo com a posição de uma slider bar, apresentam gradualmente tais informações.

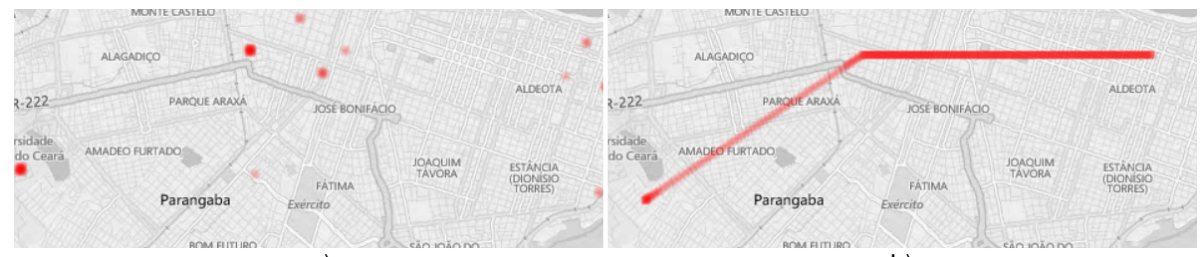

a)

b)

Figura 4. Exemplos de (a) POls e de (b) ROls obtidos automaticamente.

Em síntese, a aplicação desenvolvida utiliza o conceito de sensemaking para fornecer um caráter menos abstrato a PSTs: cada nó representa a probabilidade de um dado evento ocorrer num certo local num determinado horário e cada aresta representa possíveis mudanças de estado, estabelecidas como relações entre espaço, tempo e probabilidade. De forma complementar, considerando branching time, cada nó corresponde a um evento individual que pode originar um ou mais outros eventos distintos (nós filhos). Então, de modo geral, usando a aplicação desenvolvida para avaliar ocorrências e transições, é possível estabelecer comportamentos possíveis de indivíduos. E investigando eventos anteriores e posteriores e suas probabilidades associadas, cada um desses comportamentos possíveis pode ser então classificado ou não como comportamento provável.

\section{Experimento Realizado}

A fim de avaliar a eficiência do emprego de análise visual e sensemaking no ensino do conceito de PST, procedeu-se uma pesquisa experimental com a realização de teste prático com um total de trinta e quatro alunos, com idade média de dezenove anos, do Curso de Bacharelado em Sistemas e Mídias Digitais da Universidade Federal do Ceará, divididos em dois grupos. O primeiro grupo, de controle, contou com dezessete participantes e utilizou a aplicação desenvolvida, porém sem acesso às suas funções semiautomáticas para identificação de POIs e de ROIs. O segundo grupo, experimental, teve acesso total à ferramenta, incluindo suas funções semiautomáticas. É importante destacar que dado o caráter aplicado do trabalho, optou-se por não empregar o grupo de controle para comparação de resultados com relação a discussões entre teoria e prática; mas sim verificar se o emprego 
VII Congresso Brasileiro de Informática na Educação (CBIE 2018)

Anais do XXIX Simpósio Brasileiro de Informática na Educação (SBIE 2018)

de automatismos poderia influenciar no modo como o processo de análise visual seria desenvolvido.

Para cada grupo, o teste foi realizado durante uma aula sobre análise visual da disciplina de Matemática Aplicada a Multimídia I, com duração de 1:40 h. Essa disciplina sempre que possível emprega sensemaking no intuito de simplificar a compreensão de conceitos abstratos, particularmente algoritmos, estruturas de dados e outros assuntos relacionados à geração de interfaces gráficas, jogos eletrônicos, sistemas de visualização de dados e afins. O teste foi realizado em laboratório de informática com acesso a internet e contando com microcomputadores padrão IBM PC, executando Microsoft Windows 10, com processadores Intel i5 3470 de 3.2 GHz, 8 GB de memória RAM e monitores de 23". Para a realização do experimento foram selecionados três arquivos JSON contendo PSTs de usuários do Projeto Eai? referentes aos dias úteis entre 27/08/2015 e 01/10/2015. Durante esse período houve uma greve de professores e muitas das aulas foram suspensas. A escolha dessas datas foi particularmente importante, uma vez que ocorreram mudanças significativas na rotina da universidade, com a qual os alunos participantes do teste já poderiam estar familiarizados. Como a ocorrência da greve não foi informada durante o experimento, a tarefa de identificação dos padrões de comportamento representados tornou-se mais estimulante e desafiadora. A seguir é apresentada a sequência de etapas procedidas para a realização do experimento.

\subsection{Pré-Teste}

Antes da realização do teste, durante uma apresentação de quinze minutos, o professor da disciplina discutiu o conceito de PST, expondo os dados que as mesmas armazenam, os significados de seus nós e arestas e seus principais usos. Após essa apresentação, os alunos responderam a uma avaliação, com notas de 0,0 a 10,0, com as seguintes perguntas: O que são PSTs? Quais dados uma PST armazena? O que os nós e as arestas de uma PST representam? E para que as PSTs são utilizadas?

\subsection{Teste}

Para a realização do teste, incialmente as funções da aplicação desenvolvida foram brevemente explicadas e os alunos assinaram um termo de consentimento livre e esclarecido. Após isso, os mesmos passaram a analisar separadamente cada PST fornecida, buscando responder às seguintes perguntas: onde a pessoa reside? Qual curso a pessoa frequenta? Onde a pessoa trabalha? E quando a pessoa vai ao restaurante universitário? Devido à política de privacidade do Projeto Eaí? e às características probabilísticas das PSTs, o teste não se preocupou com a resolução formal das quatro questões elencadas. O intuito principal foi, na verdade, avaliar se os participantes seriam capazes de apresentar respostas consistentes, estabelecendo hipóteses e utilizando a aplicação desenvolvida para confirmá-las ou refutá-las, de acordo com os princípios da análise visual [Fabrikant and Lobben 2009]. Dado o acesso à internet do laboratório utilizado, os participantes puderam utilizar, paralelamente à realização do teste, serviços online como visualização de mapas e consultas a endereços, dentre outros. Os alunos responderam as quatro perguntas num questionário específico, sempre explicitando e justificando suas observações, incluindo hipóteses estabelecidas e como as mesmas foram avaliadas. Além disso, os participantes também anotaram, nos próprios questionários, suas observações gerais sobre o uso da aplicação desenvolvida e o modo como cada resposta foi obtida, incluindo os serviços online utilizados. 
VII Congresso Brasileiro de Informática na Educação (CBIE 2018)

Anais do XXIX Simpósio Brasileiro de Informática na Educação (SBIE 2018)

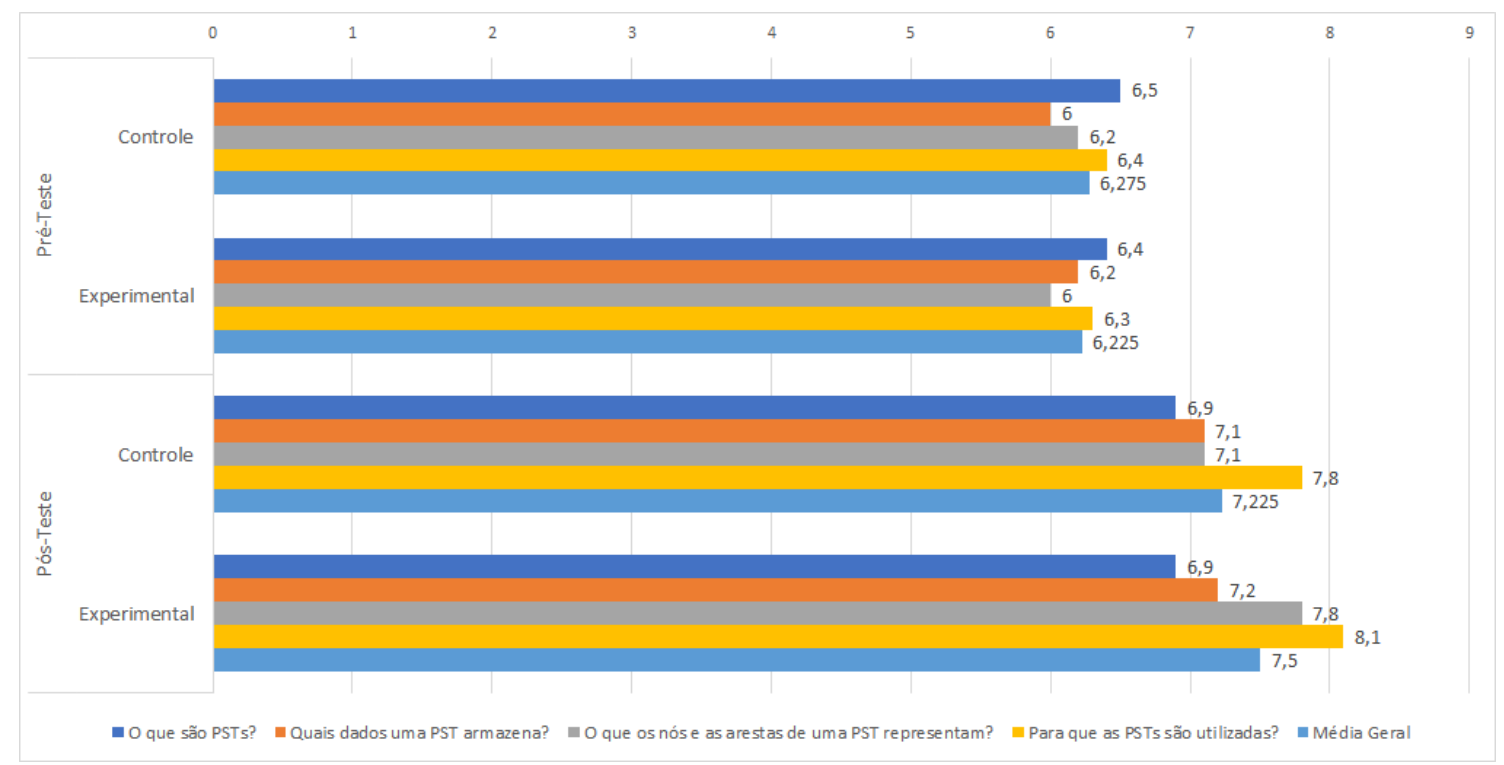

Figura 5. Notas médias (entre 0,0 e 10,0) das avaliações realizadas pelos grupos de controle e experimental durante as etapas de pré-teste e de pós-teste.

\subsection{Pós-Teste}

Após a realização do teste, os participantes responderam novamente à mesma avaliação apresentada na etapa de pré-teste para fins de análise da eficiência do uso da aplicação desenvolvida.

\section{Análise de Resultados}

A seguir são apresentadas análises quantitativas e qualitativas referentes aos resultados do experimento realizado.

\subsection{Análise Quantitativa}

Do ponto de vista quantitativo, observa-se que as notas das avaliações na etapa de pósteste apresentaram incremento em relação à de pré-teste (Figura 5), com aumento de 0,95 na média geral para o grupo de controle e 1,275 para o experimental, com um leve ganho para o último. Tais acréscimos já eram esperados, uma vez que todos os participantes praticaram o uso das PST através da aplicação desenvolvida, o que provavelmente influenciou na resposta à segunda aplicação da avaliaçao, na fase de pós-teste [Brown et al. 1989]. Isso pode ser particularmente notado na questão sobre para que as PSTs são utilizadas, onde ocorreu um ganho de 1,4 pontos para o grupo de controle e 1,8 para o experimental. Percebe-se também que o grupo experimental obteve um aumento substancial $(1,8$ pontos) em comparação com o grupo de controle ( 0,9 pontos) na questão sobre o que representam nós e arestas de PSTs. Tal diferença pode advir do acesso diferenciado dos grupos às funções semiautomáticas para identificação de POIs e de ROIs.

Com relação à quantidade total de respostas válidas (Figura 6), adequadas aos dados das PSTs analisadas e devidamente justificadas, nota-se que quase não houve diferenças significativas entre os grupos nos questionários da etapa de teste. A única exceção, porém, refere-se ao local de trabalho do usuário da segunda PST avaliada: das dezessete respostas válidas possíveis (quantidade total de participantes em cada grupo), o 
VII Congresso Brasileiro de Informática na Educação (CBIE 2018)

Anais do XXIX Simpósio Brasileiro de Informática na Educação (SBIE 2018)

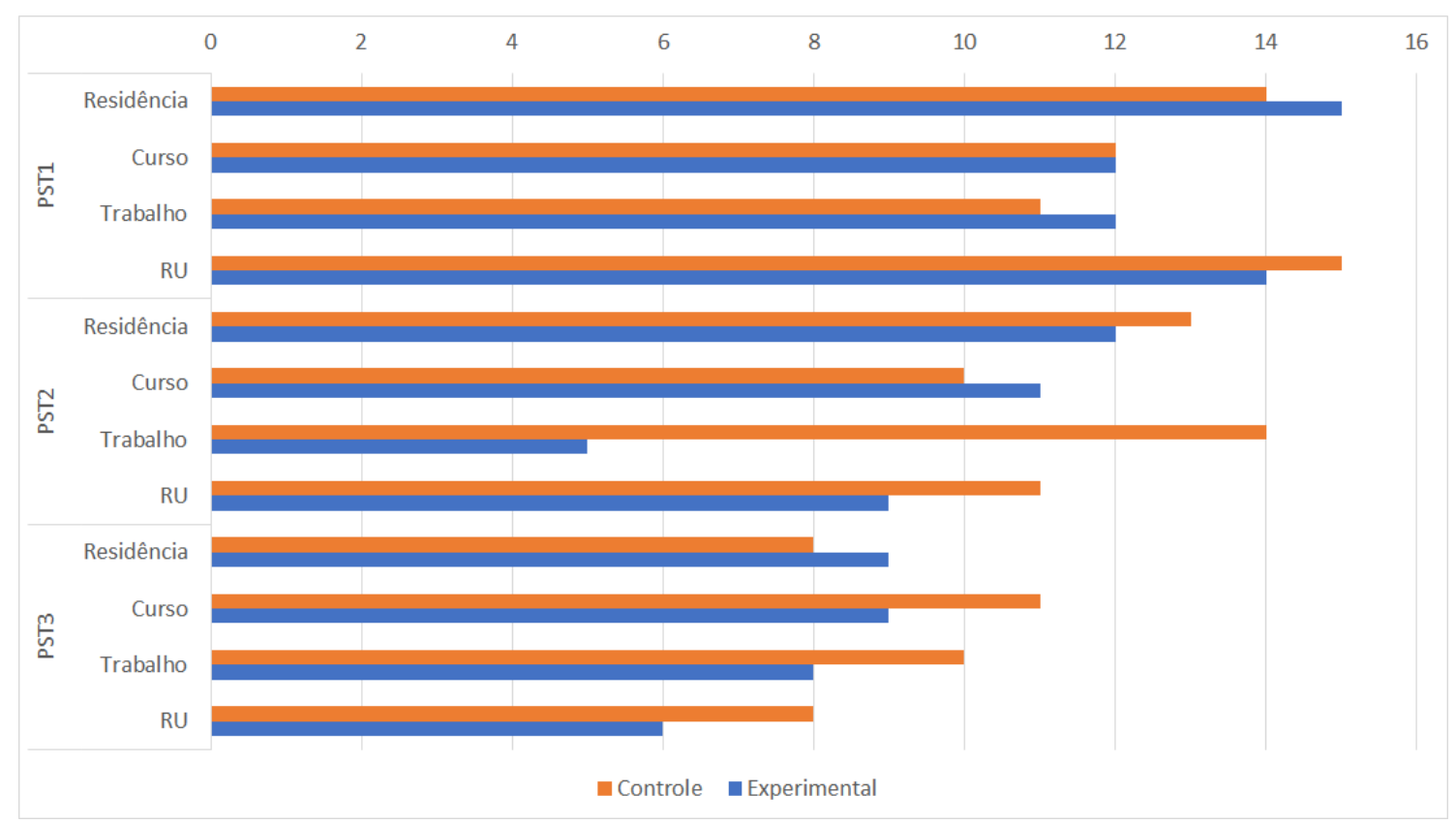

Figura 6. Quantidades de respostas válidas nos questionários da etapa de teste.

grupo de controle forneceu somente cinco e já o grupo experimental, quatorze. Mais uma vez as ferramentas semiautomáticas podem ter influenciado o desempenho dos grupos.

\subsection{Análise Qualitativa}

Avaliando as respostas e anotações dos questionários da etapa de teste, é importante destacar que, independente de pertencerem ao grupo de controle ou ao grupo experimental, os participantes do experimento estabeleceram hipóteses bem fundamentadas a fim de confirmá-las ou refutá-las posteriormente. Por exemplo, apesar de não perguntado, diversos participantes indicaram que o primeiro e o segundo usuários das PSTs analisadas frequentaram locais de culto religioso. Da mesma forma, foram apontados alguns dos prováveis locais mais frequentados: uma padaria para o primeiro usuário, um shopping para o segundo e uma academia de ginástica para o terceiro. Também foram indicadas as possíveis paradas de ônibus geralmente utilizadas pelo segundo e pelo terceiro usuários e que o primeiro usuário não frequentou a universidade durante o período avaliado. Houve ainda observações mais específicas, como a possibilidade de o terceiro usuário ser um aluno novato na cidade, já que foi identificada uma viagem do mesmo ao Estado de Alagoas logo no início do período considerado e que ele frequentou diversos pontos turísticos próximos à orla marítima, inclusive à noite. Investigando a mesma situação, um participante ainda observou possíveis erros de GPS capturados na PST desse mesmo usuário, pois havia pontos de presença em pleno mar, a cerca de cem metros da costa. Segundo esse participante, e devido à sua experiência própria, como aquele local específico não era adequado à prática de surfe ou outros esportes aquáticos, provavelmente houve algum tipo de inconsistência nos dados representados. Vale destacar que tal tipo de avaliação, que considera as experiências prévias dos analistas, são um diferencial particularmente importante da análise visual em comparação a outras técnicas de avaliação, como visualização científica ou aquelas baseadas em machine learning.

No geral, os participantes do experimento indicaram corretamente possíveis lo- 
VII Congresso Brasileiro de Informática na Educação (CBIE 2018)

Anais do XXIX Simpósio Brasileiro de Informática na Educação (SBIE 2018)

cais de residência para os três usuários, muitas vezes incluindo respectivos logradouros e números. No entanto, a não informação de que os dados se referiam a uma época de greve gerou muitas dúvidas sobre o curso frequentado pelo primeiro usuário, que realmente não compareceu à universidade no período avaliado. De todo modo, diversos participantes do teste apontaram que ou o usuário não frequentou um curso ou que o mesmo era um aluno semipresencial, sendo ambas respostas válidas. Com relação aos prováveis locais de trabalho, muitos participantes observaram que o primeiro usuário mantinha uma vida noturna bastante ativa nos arredores de sua residência, o que levou a possibilidades de profissões bastante diversas, todas devidamente justificadas, abrangendo desde celebrante de cerimônias religiosas, no local de culto anteriormente identificado, e vigilante noturno a traficante de drogas. Ainda no que se refere ao local de trabalho, vários participantes do grupo experimental, que tiveram acesso às ferramentas de POIs e de ROIs, apontaram que o segundo usuário provavelmente trabalhava numa universidade particular. $\mathrm{O}$ mesmo não aconteceu com os participantes do grupo de controle, o que mais uma vez pode justificar as diferenças de ganhos discutidas anteriormente na avaliação quantitativa do experimento. No que se refere às idas ao restaurante universitário, é importante destacar que as PSTs analisados apontavam que todos os três usuários praticamente não frequentaram o local. Porém, alguns participantes do grupo experimental encontraram um padrão que indicava que o segundo usuário almoçou algumas vezes numa lanchonete na universidade. Tal descoberta, no entanto, provavelmente só foi possível novamente graças ao uso das ferramentas semiautomáticas disponibilizadas. Por fim, observou-se que o tempo médio gasto pelos membros do grupo de controle para analisar cada PST foi de vinte minutos, enquanto o grupo experimental demandou um tempo menor para a tarefa, de cerca de quinze minutos. Mais uma vez, o uso das ferramentas semiautomáticas pode ajudar na explicação de tal diferença no tempo necessário para as análises.

\section{Considerações Finais}

Com base nos resultados apresentados nesse trabalho, percebe-se que o emprego de análise visual, sensemaking e branching time no ensino de PSTs mostrou-se como uma alternativa educacional viável. De um modo geral, houve ganho na apresentação de conceitos com base no uso da aplicação desenvolvida e, particularmente, as análises quantitativa e qualitativa evidenciaram que o acesso às ferramentas semiautomáticas pode ter influenciado significativamente as respostas dos participantes do teste realizado. Como trabalhos futuros, novos experimentos deverão ser realizados, abordando o ensino de outras estruturas de dados e empregando novas aplicações específicas. Com isso, espera-se estar contribuindo para a criação de ferramentas mais aplicadas para o ensino não só de PSTs, mas também de outros conceitos considerados demasiadamente abstratos, buscando assim tornar a educação mais prática e efetiva.

\section{Referências}

Aigner, W., Miksch, S., Müller, W., Schumann, H., and Tominski, C. (2008). Visual methods for analyzing time-oriented data. IEEE transactions on visualization and computer graphics, 14(1):47-60.

Beheshti, E., Villanosa, K., and Horn, M. (2018). Understanding parent-child sensemaking around interactive museum exhibits. Annual Meeting of the American Educational Research Association (AERA 2018). 
VII Congresso Brasileiro de Informática na Educação (CBIE 2018)

Anais do XXIX Simpósio Brasileiro de Informática na Educação (SBIE 2018)

Blackwell, A. F. (2006). The reification of metaphor as a design tool. ACM Transactions on Computer-Human Interaction (TOCHI), 13(4):490-530.

Bobis, J. and Way, J. (2018). Building connections between children's representations and their conceptual development in mathematics. In Forging Connections in Early Mathematics Teaching and Learning, pages 55-72. Springer.

Brown, J. S., Collins, A., and Duguid, P. (1989). Situated cognition and the culture of learning. Educational researcher, 18(1):32-42.

Caglayan, G. (2018). Coordinating analytic and visual approaches: Math majors' understanding of orthogonal hermite polynomials in the inner product space pnp in a technology-assisted learning environment. The Journal of Mathematical Behavior.

Dervin, B. (1983). An overview of sense-making research: Concepts, methods, and results to date. The Author.

Fabrikant, S. I. and Lobben, A. (2009). Introduction: Cognitive issues in geographic information visualization. Cartographica: The International Journal for Geographic Information and Geovisualization, 44(3):139-143.

Keim, D., Andrienko, G., Fekete, J.-D., Görg, C., Kohlhammer, J., and Melançon, G. (2008). Visual analytics: Definition, process, and challenges. In Information visualization, pages 154-175. Springer.

Leite, A. J. M., Santos, E., Vidal, C. A., and De Macêdo, J. A. F. (2017). Visual analysis of predictive suffix trees for discovering movement patterns and behaviors. In 2017 30th SIBGRAPI Conference on Graphics, Patterns and Images (SIBGRAPI), pages 103-110. IEEE.

Martínez, M. (2011). Travelling in branching time. Disputatio, 4(31):271-287.

Ng, O.-L., Sinclair, N., and Davis, B. (2018). Drawing off the page: How new 3d technologies provide insight into cognitive and pedagogical assumptions about mathematics. The Mathematics Enthusiast, 15(3):563-578.

Rocha, C. L., Brilhante, I. R., Lettich, F., De Macedo, J. A. F., Raffaetà, A., Andrade, R., and Orlando, S. (2016). Tpred: a spatio-temporal location predictor framework. In Proceedings of the 20th International Database Engineering \& Applications Symposium, pages 34-42. ACM.

Ron, D., Singer, Y., and Tishby, N. (1994). Learning probabilistic automata with variable memory length. In Proceedings of the seventh annual conference on Computational learning theory, pages 35-46. ACM.

Russell, D. M., Stefik, M. J., Pirolli, P., and Card, S. K. (1993). The cost structure of sensemaking. In Proceedings of the INTERACT'93 and CHI'93 conference on Human factors in computing systems, pages 269-276. ACM.

Siemens, G. and Long, P. (2011). Penetrating the fog: Analytics in learning and education. EDUCAUSE review, 46(5):30.

Warburton, E. C. and Laughlin, G. (2018). A performed solution to the pythagorean problem: The three bodies project. Leonardo, pages 1-12. 